\title{
Advanced Monitoring of R-22 Refrigerant Leakage
}

\author{
Mohan $\mathbf{M}^{1}$, Vidyadhara B.V ${ }^{1}$, Vishesh $\mathbf{S}^{2}$, Manu Srinath ${ }^{2}$ \\ Student, Department of Mechanical Engineering, RV College of Engineering, Bengaluru, India ${ }^{1}$ \\ B.E, Department of Telecommunication Engineering, BNM Institute of Technology, Bengaluru, India ${ }^{2}$
}

\begin{abstract}
Chlorodifluoromethane and difluoromonochloromethane is a Hydro-Fluoro-Chloro-Carbon (HCFC). It is a colourless gas and is better known as HCFC-22 or R-22. It is used as a refrigerant in medium and low temperature commercial and industrial refrigeration and freezer rooms, and also in cold stores, cabinets, water chillers and process cooling. R-22 is often used as an alternative to the highly ozone depleting R-11 and R-12 because of its relatively low ozone depletion potential of 0.055 among the lowest of chlorine containing halo-alkanes. In this paper, we propose a system to detect the leakage of R-22 in domestic and industrial refrigeration applications. We have proposed a low cost, portable and wireless detection system using android application and automated responses to cease the leakage of refrigerant.
\end{abstract}

Keywords: Chlorodifluoromethane, difluoromonochloromethane, Hydro-Fluoro-Chloro-Carbon (HCFC), HCFC-22, R-22, R-11, R-12, halo-alkanes, portable, wireless detection system, android application, automated responses.

\section{INTRODUCTION}

Refrigerants are specially selected substances that have The clean air act of 1990 mandated the termination of certain chemical characteristics including good production of all CFCs such as R-12 in the USA by the refrigeration performance, low flammability and toxicity, year 2000. [5][6] Government data indicate that that the compatibility with compressor lubricating oils and metals, structural difference between them, R-12 has twenty times and good heat transfer characteristics. [1] They are usually the ozone depletion potential in the upper atmosphere than identified by a number that relates to their molecular R-22. Nevertheless, R-22 and other HCFCs are also composition. The ASHRAE handbook identifies a large scheduled by the law for phase out of production by the number of refrigerants by number. Inorganic refrigerants year 2030. [7] are designated by 700 , plus their molecular weight.

Figure 1 shows the representation of a refrigerant. [2] The important examples are refrigerants R-12 and R-22. R-12, dichlorodifluoromethane, has two fluorine, one carbon, and two chlorine atoms in a methane-type structure. Chlorine and fluorine, replace hydrogen atoms in the $\mathrm{CH}_{4}$ molecular structure. R-22 monochlorodifluoromethane, has a similar structure to R-12, except for a single hydrogen atom replacing a chlorine atom. The commonly used CFCs and HCFCs are a cause for great concern, because their accumulation in the upper atmosphere creates a hole in the ozone layer that normally shields the earth from the UV radiations of the sun. [3]

In 1987, more than 35 countries, including the United States of America, signed the Montreal Protocol on substances that deplete the ozone layer. [4] The Montreal Protocol called for a freeze in 1989 and reductions in the 1990s on the production levels of R-11, R-12, R-113, R114 and R115.

\section{REFRIGERATION CYCLE}

According to the second law of thermodynamics, heat cannot flow from a colder region to a hotter region spontaneously; work is required to achieve it. The processes that take place during refrigeration and the respective thermodynamics involved are called the refrigeration cycle. Figure 2 shows the schematic diagram of a refrigeration unit and figure 3 shows the processes that take place during the cycle.

Figure 2 shows the components of a refrigeration unit. It mainly consists of:

- Compressor

- Condenser

- Expansion Valve

- Evaporator

A. Compressor

The vapour refrigerant from the evaporator is supplied to The HCFCs, lacking chlorine, are no threat to ozone layer the compressor where the pressure of the vapour but are not in common usage as refrigerants. CFCs which refrigerant increases to $\mathrm{P}_{2}$ (compressor delivery pressure) contain more chlorine than HCFCs, are more serious from $\mathrm{P}_{1}$ (evaporator exit pressure). Also the temperature or offenders, are very stable and do not breakdown rapidly in the lower atmosphere.

the vapour refrigerant increases from $T_{1}$ to $T_{2}, T_{2}$ is the superheated level. The whole process is assumed to be an 
International Journal of Advanced Research in Computer and Communication Engineering ISO 3297:2007 Certified

Vol. 6, Issue 1, January 2017

isentropic process, where entropy of the vapour refrigerant whole process takes place at constant pressure, hence remains constant. called isobaric heat rejection process, i.e, $\mathrm{P}_{2}=\mathrm{P}_{3}$.

$\mathrm{P}_{1} / \mathrm{P}_{2}=$ Constant, for a given evaporative load.

C. Expansion Valve

B. Condenser

The high pressure liquid refrigerant passes through the expansion valve where in the pressure drops to The high pressure superheated vapour is made to pass evaporative pressure, i.e from $\mathrm{P}_{3}$ to $\mathrm{P}_{4}$, where $\mathrm{P}_{3}$ is through condenser coils which transfers the heat to the expansion valve inlet pressure and $\mathrm{P}_{4}$ is evaporative ambient and condenses the vapour to liquid state. The pressure.

\section{$\mathrm{R}-\mathrm{XYZ}$}

\section{$R=$ Refrigerant}

$X=$ one less than the number of carbon atoms in the compound $Y=$ number of hydrogen atoms +1 $Z=$ number of fluorine atoms

Figure 1 Representation of a refrigerant

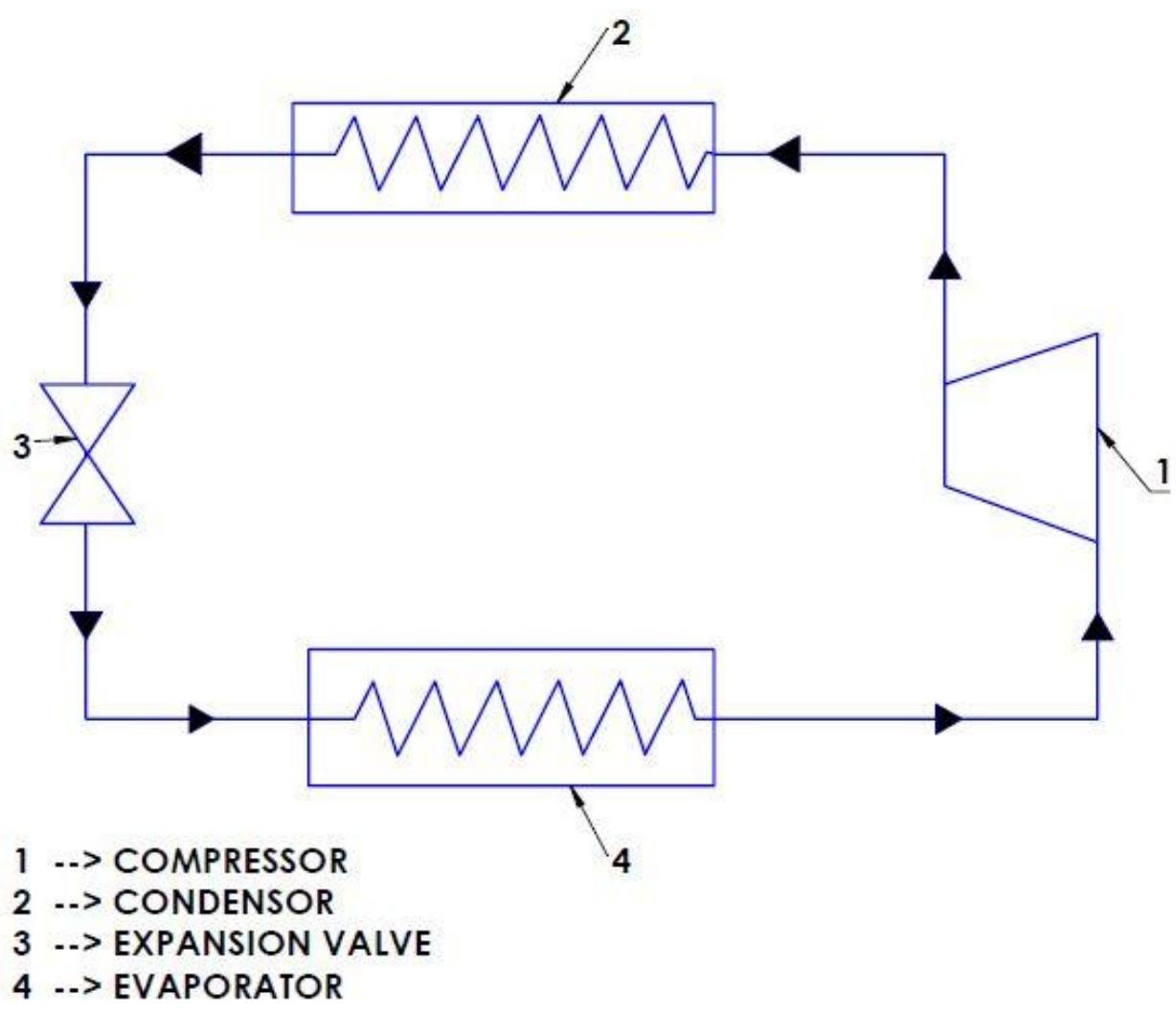

Figure 2 Refrigeration Cycle 

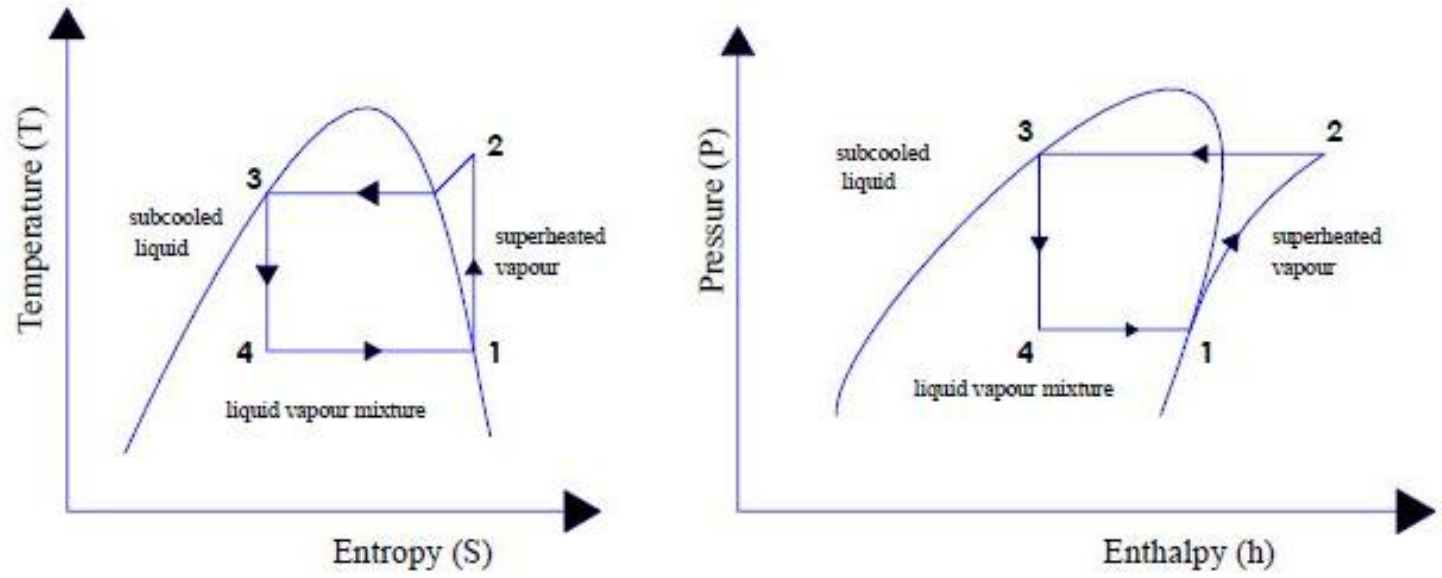

PROCESSES:

$1-2$ : ISENTROPIC COMPRESSION

$2-3$ : ISOBARIC HEAT REJECTION

$3-4$ : ISENTROPIC EXPANSION

4-1: ISOBARIC HEAT ADDITION

Figure 3 Processes that take place during refrigeration cycle

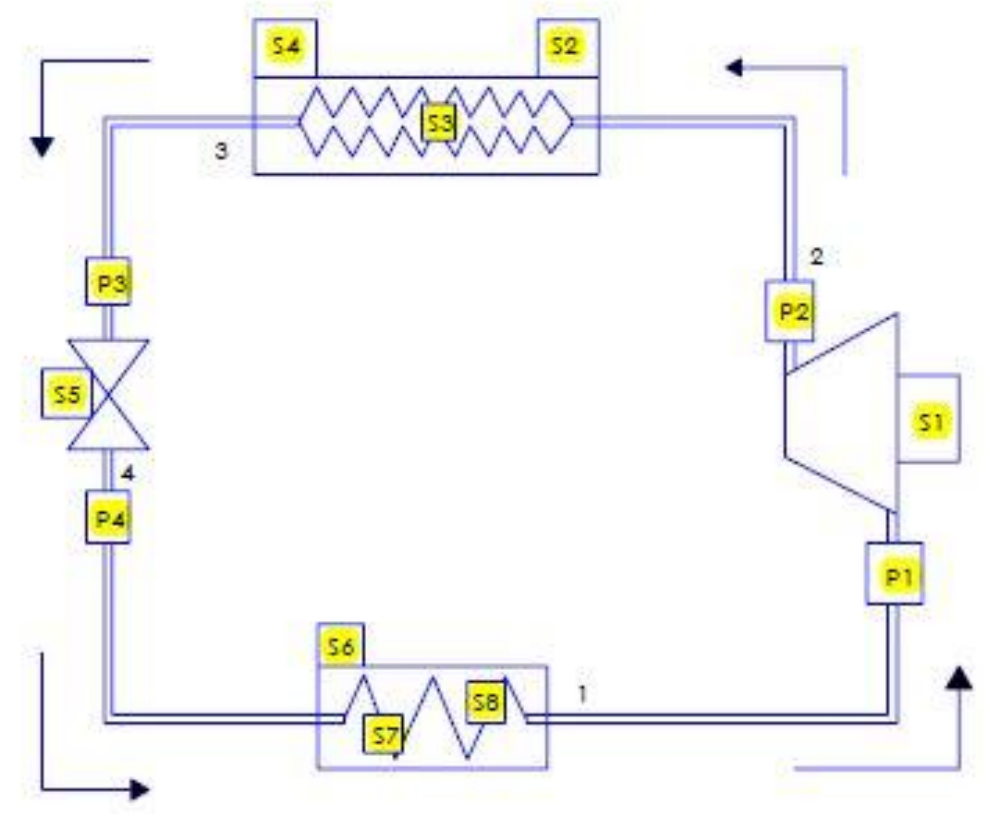

1) LOW PRESSURE HIGH TEMPERATURE VAPOUR

2) HIGH PRESSURE HIGH TEMPERATURE SUPERHEATED VAPOUR

3) HIGH PRESSURE LOW TEMPERATURE LIQUID

4) LOW PRESSURE LOW TEMPERATURE LIQUID

\section{P1, P2, P3, P4 - PRESSURE SENSORS}

S1, S2, S3, S4, S5, S6, S7, S8 - ppm SENSORS

Figure 4 Block diagram of the refrigeration system and the sensors used to detect any leakage of refrigerant 
Vol. 6, Issue 1, January 2017

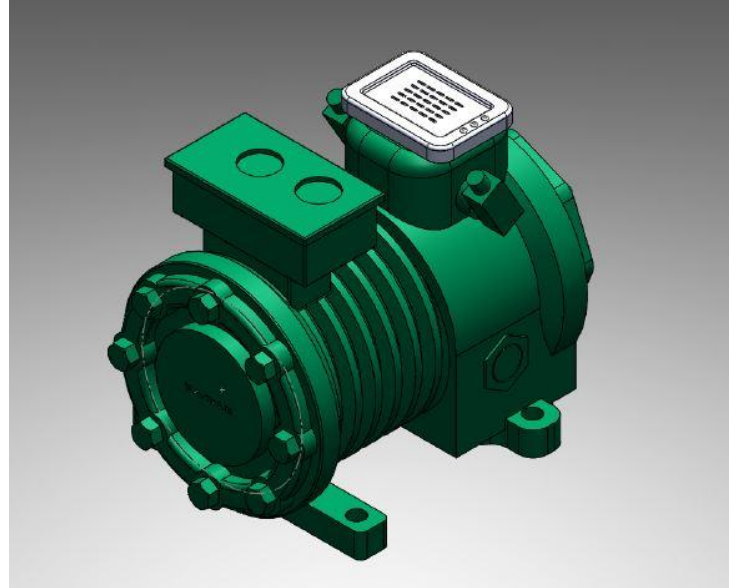

Figure 5 3-D Model of Compressor

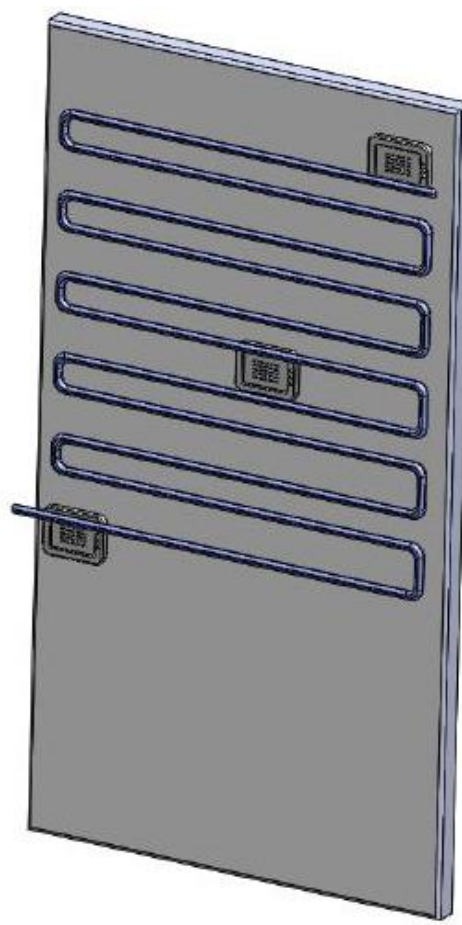

Figure 6 Position of sensors on the condenser pipes

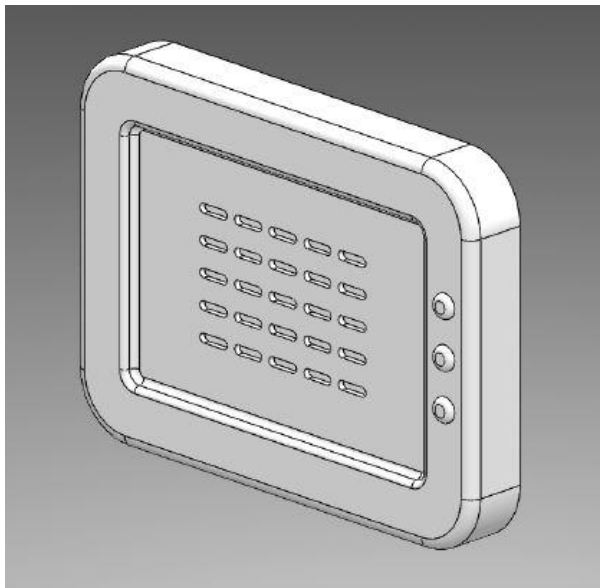

Figure 7 3-D model of the refrigerant leakage detection sensor

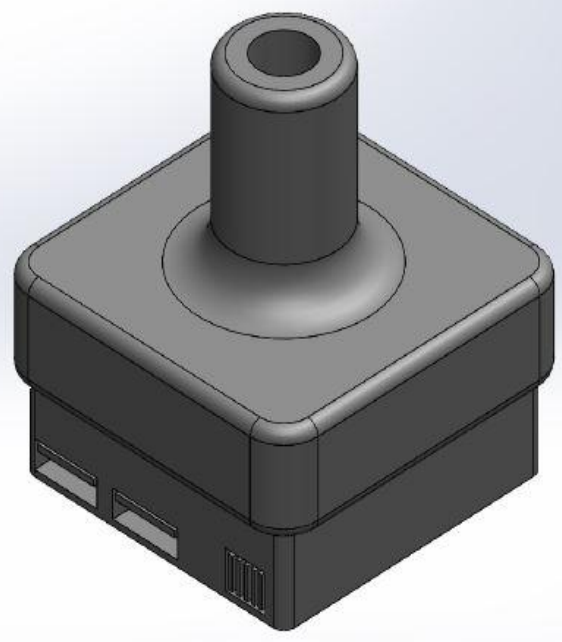

Figure 8 Pressure sensors equipped within the pipes

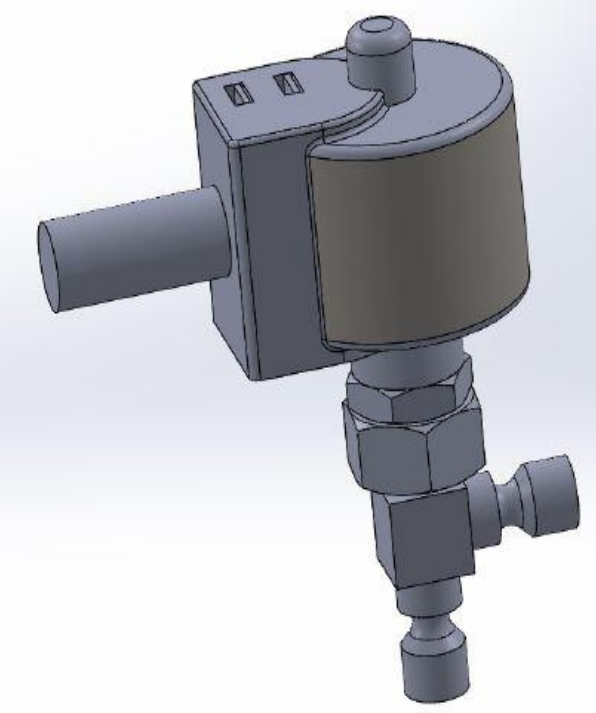

Figure 9 Electronic Valve

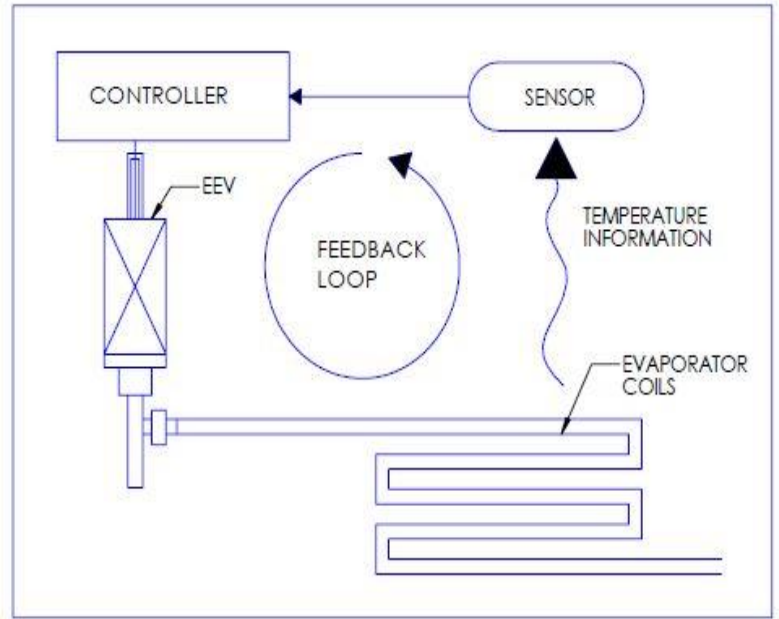

Figure 10 Feedback loop 
Vol. 6, Issue 1, January 2017

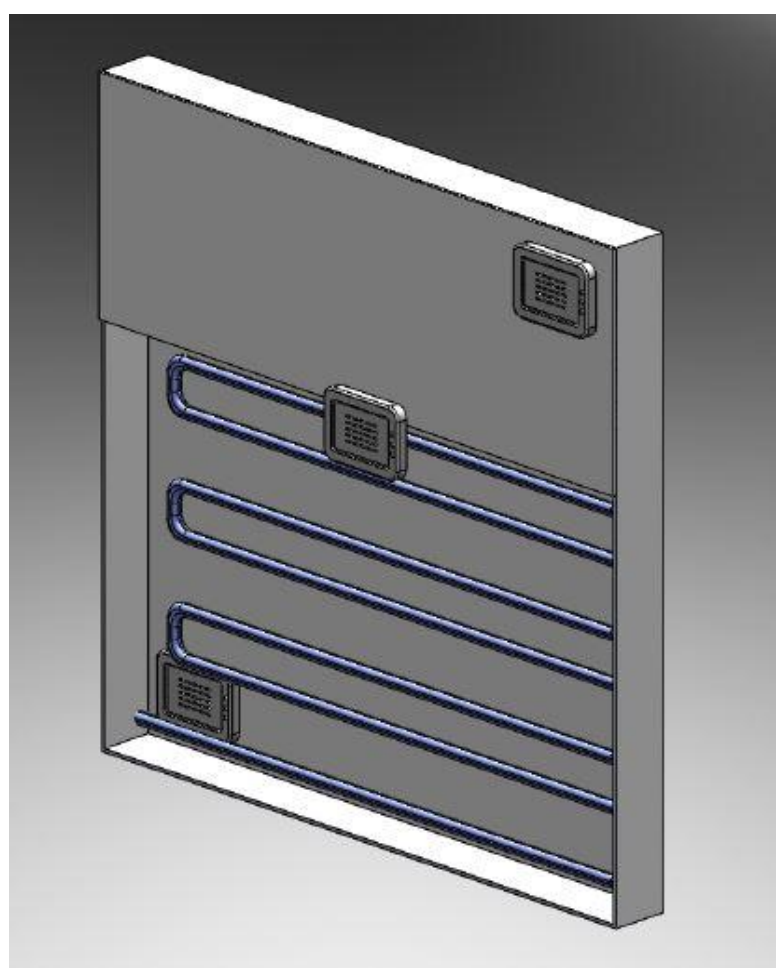

Figure 11 Evaporator

The whole process occurs at constant entropy, hence called isentropic expansion process.

\section{Evaporator}

The liquid refrigerant enters the evaporator (evaporative coils), the entropy of the liquid refrigerant increases, thus the refrigerant vaporises at a constant pressure to become vaporised refrigerant. The whole heat addition process occurs at constant pressure, hence called isobaric heat addition process, i.e $\mathrm{P}_{1}=\mathrm{P}_{4}$.

\section{III.METHODOLOGY}

Figure 4 shows the block diagram of the refrigeration system and the sensors used to detect any leakage of refrigerant. As soon as the process or system is initiated, the compressor is switched on and expansion valve opens depending on the evaporator load. The "low pressure high temperature" refrigerant enters the compressor and the compressor delivers "high pressure high temperature superheated" vapour, i.e from state 1 to state 2 as mentioned in the block diagram. Figure 5 shows the 3D model of a compressor. The pressure sensors $\mathrm{P}_{1}$ and $\mathrm{P}_{2}$ are placed at inlet and outlet of the compressor as shown in figure 4. The compressor ratio $\mathrm{P}_{2} / \mathrm{P}_{1}$ is almost a constant for a given evaporative load. Thus any change or variation in compressor ratio is processed by the microcontroller and displayed suitably. The sensor $S_{1}$ is placed on or near the compressor to detect the leakage if any and a signal is passed to the microcontroller for processing.

Now, the "high pressure high temperature superheated" refrigerant is made to pass through condenser where the refrigerant gets condensed to a lower temperature liquid state, i.e, from state 2 to state 3 as shown in figure 4 . The sensors $S_{2}, S_{3}$ and $S_{4}$ are placed near the condenser pipes as shown in figure 6 . Figure 7 shows the 3D model of a refrigerant leakage detection sensor. The sensor has the following features:

- High measuring range; i.e 0-10,000 ppm

- High accuracy

- Robust in nature

- Simple in design

- Easily connectable to microcontroller for processing

- $\mathrm{ADC}$ unit to convert real time analog values to digital for processing by ALU of the microcontroller.

Figure 8 shows the pressure sensors equipped within the pipes as shown in figure 4 . The sensor senses the pressure and is sent to the microcontroller, where the pressure ratio is checked for the nominal pressures depending on evaporative load. If there is any leakage, there is pressure change in the pressure ratio and thus is identified by the microcontroller.

The "high pressure low temperature liquid" refrigerant from the condensing coil enters the throttle valve at constant temperature, where "high pressure" liquid refrigerant gets throttled to low pressure liquid refrigerant in the electronic expansion valve as shown in figure 9 i.e., state 3 to state 4 as shown in figure 4 . The temperature of the liquid refrigerant at the exit of the electronic expansion valve is lower than the evaporator temperature. The pressure sensors $\mathrm{P}_{3}$ and $\mathrm{P}_{4}$ are placed at the inlet and the outlet of the expansion valve as shown in figure 4 . The pressure ratio $\mathrm{P}_{3} / \mathrm{P}_{4}$ is almost a constant for a given evaporative load that is maintained by the feedback loop as shown in figure 10 . The sensor $S_{5}$ placed near the electronic expansion valve, detects leakage of refrigerant as shown in figure 4. Thus, the "Low temperature low pressure liquid" refrigerant is made to pass through the evaporator which is as shown in figure 11. Here the liquid temperature gains the heat from the evaporative space and becomes "Low temperature high pressure vapour" refrigerant i.e., from state 4 to state 1 which is as shown in figure 4.

\section{A. Processing and Output}

Figure 12 shows the circuit diagram of a microcontroller interfaced to a GSM module, indicator LEDs and Smartphone interfaced via Bluetooth. [9]

Signals, P1, P2, P3 and P4 are connected to I/O ports A0, $\mathrm{A} 1, \mathrm{~A} 2$ and A3 respectively of the microcontroller. Signals, S1, S2, S3, S4, S5, S6, S7 and S8 are connected ot A4, A5, A6, A7, A8, A9, A10, A11 and A12 respectively on the microcontroller.

A software program is written and dumped into the memory of the microcontroller. There are two challenges to be addressed by the microcontroller, they are:

- Amount of Leakage (in ppm) - the ppm sensors/ leakage detection sensors senses the leakage of R22 and the values are fed to the microcontroller.

- Region of leakage 
There are three conditions:

- If the amount of leakage detected is $<1 \mathrm{ppm}$, then the green LED glows on indicating normal functioning.

- If the amount of leakage detected in between $1 \mathrm{ppm}$ and $10 \mathrm{ppm}$, then a yellow LED glows indicating minimal leakage.

- If the amount of leakage exceeds 10 ppm, the red LED glows indicating maximum hazardous level of leakage.

Similarly,

- P2/P1 value is compared with the standard values and LEDs glow accordingly.

- P3/P4 value is compared with standard values and LEDs glow accordingly

Figure 14 shows the flow of signals in and out of the microcontroller.

Figure 13 shows the sensor parameters and pressure parameters with their digital representation and regions where leakage occurs.

\section{IV.EXPECTED RESULTS}

As mentioned earlier, any leakage of the refrigerant R-22 in the refrigeration system can be indicated in 3 ways:

- Indication from LEDs

Android application

GSM module [10]

\section{A. LEDs}

Figure 15 shows the leakage detection of the refrigerant at particular region of the refrigerating system. A LED panel is fixed on the door panel of the refrigerator and it will indicate any leakage and the amount of leakage.

If the LED1 glows, then there is a leakage of the refrigerant at the compressor, the amount of leakage is colour coded as shown in figure 15.

Similarly, if LED2, LED3 and LED4 glow, then it indicates at the condenser, EEV or evaporator respectively.

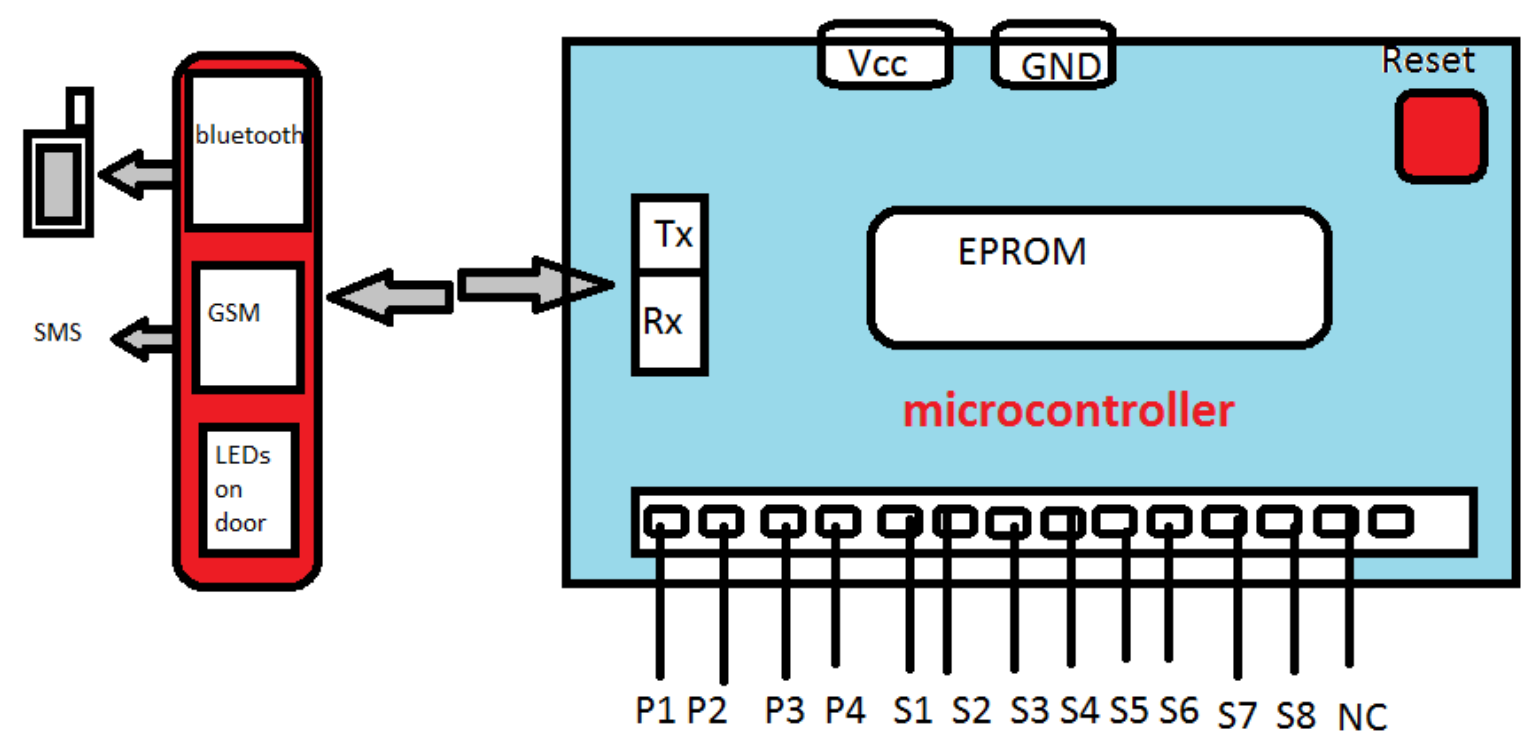

Figure 12 Circuit diagram of a microcontroller interfaced to a GSM module, indicator LEDs and Smartphone interfaced via Bluetooth

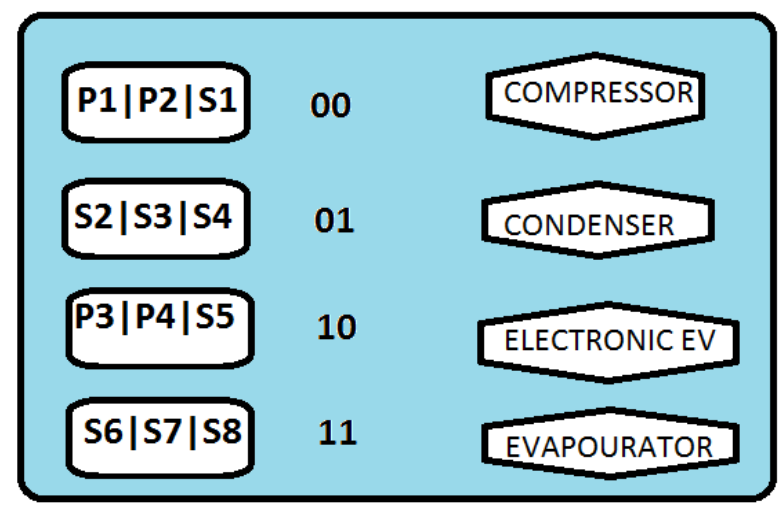

Figure 13 Sensor parameters and pressure parameters with their digital representation and regions where leakage occurs 


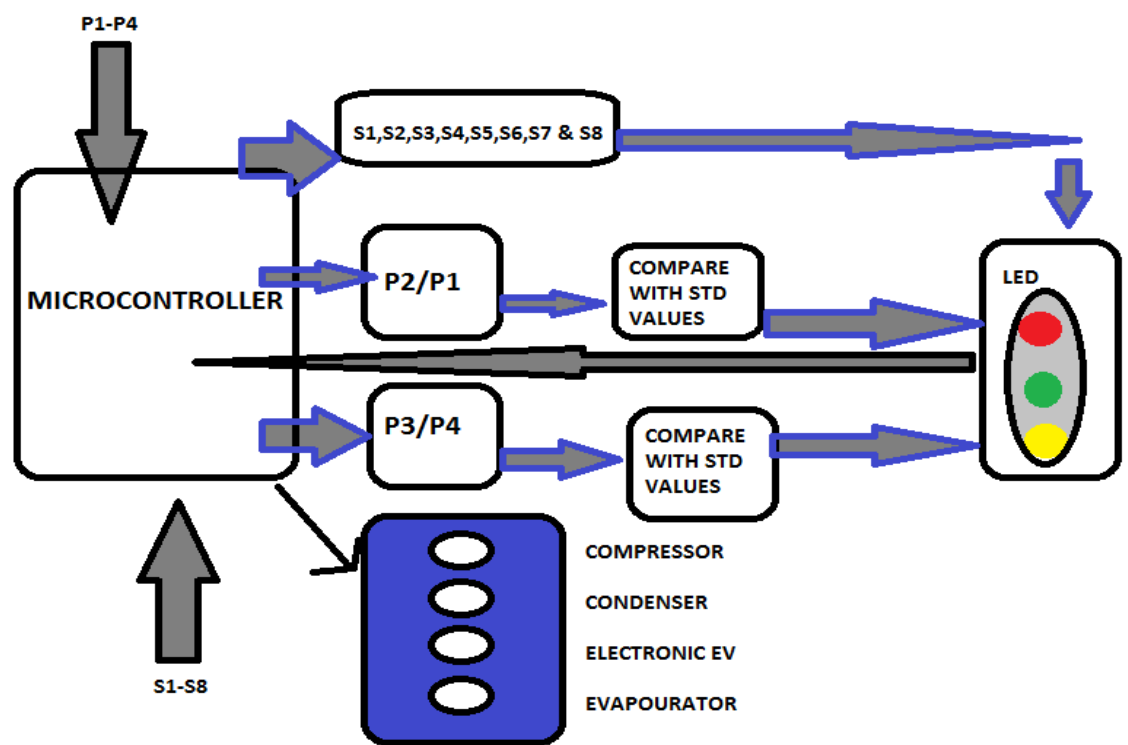

Figure 14 Flow of signals in and out of the microcontroller

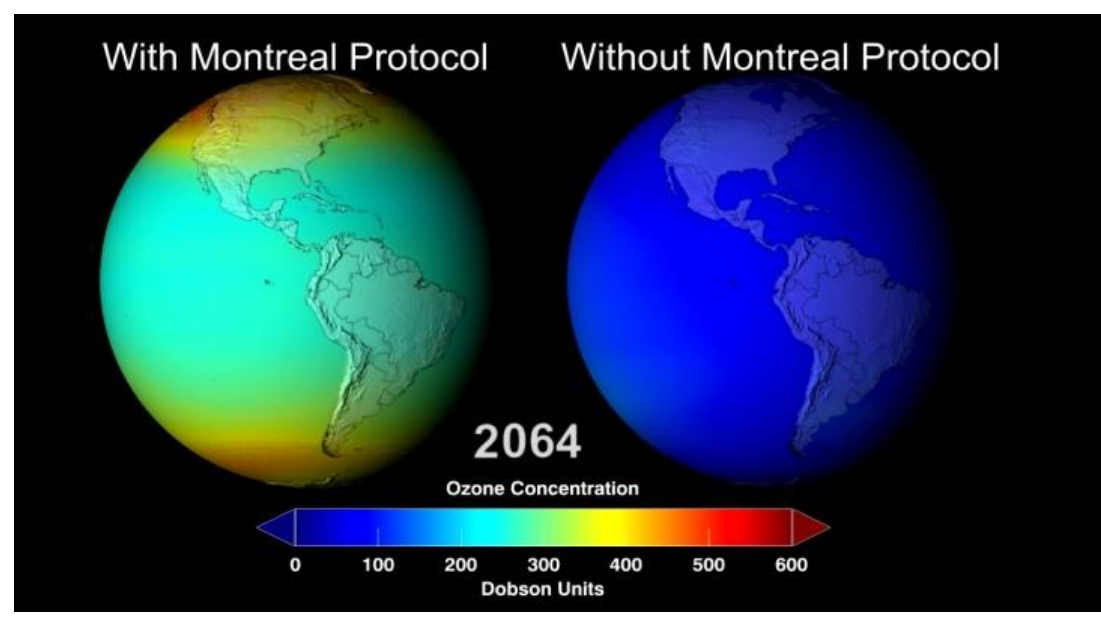

Figure 17
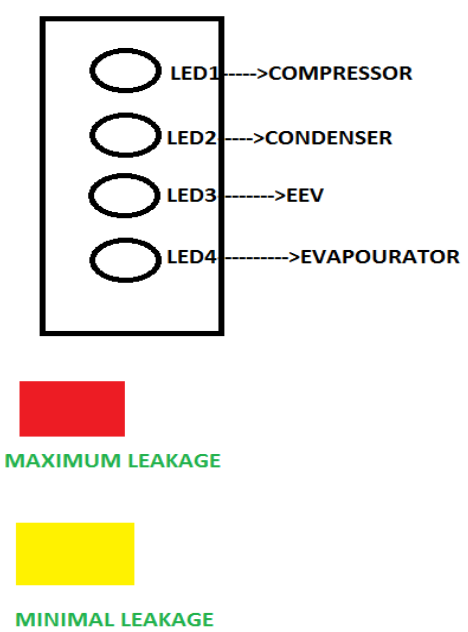

NORMAL FUNCTIONING

Figure 15 Leakage detection of the refrigerant at particular region of the refrigerating system 
Vol. 6, Issue 1, January 2017

\section{B. Android Application}

An android Smartphone application is developed and is installed in a compatible Smartphone. Any leakage during the refrigeration process is detected by the sensors and the signal is passed through the Smartphone via Bluetooth and any refrigerant leakage can be easily monitored using the Smartphone.

Also, provisions are made to stop the refrigeration process immediately in case of any leakage in order to prevent further leakage of the refrigerant. Figure 16 shows the layout of the android application for leakage detection.

\section{GSM Module}

If the refrigerant leakage has reached dangerous levels and in case the device used to monitor the leakage is not within the range of the Bluetooth, then there is a provision to send SMS to a designated mobile number. Also, any failure in the hardware, power supply, etc, can be notified by sending SMS using the GSM module.

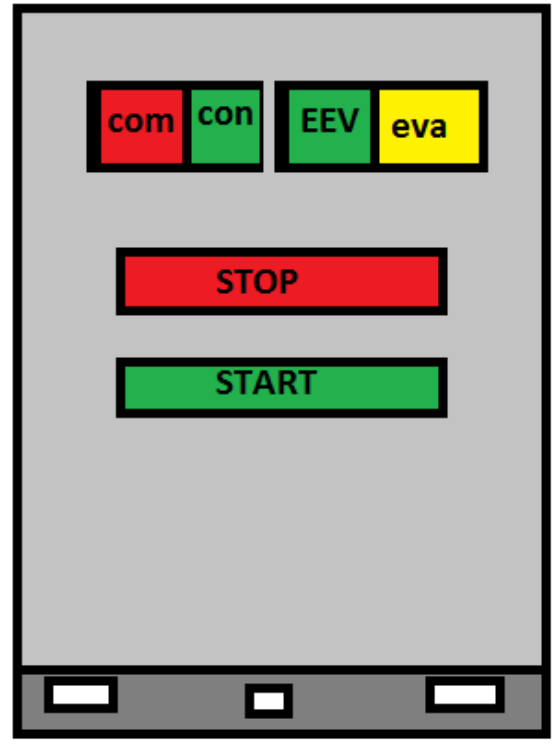

Figure 16 Layout of the android application for leakage detection

\section{DISCUSSIONS}

The Montreal Protocol actually restricts "consumption" rather than use of ozone-depleting substances, but it defines consumption as production plus imports less exports and tightly controlled, exempted uses. By addressing production and international trade rather than use, the Protocol allows each country to determine whether and how to allocate both the limited amounts by application and the production quotas by manufacturer.

The Protocol placed a limit, often referred to as cap, on HCFC production (actually "consumption" as defined) beginning in 1996 in developed countries. The cap equals $2.8 \%$ of CFC plus $100 \%$ of HCFC "consumption" in 1989 , both weighted by the ozone-depletion potentials (ODPs) of the individual substances. Further reduction steps will follow. Those steps will limit "consumption" to $65 \%$ of the cap by $2004,35 \%$ by $2010,10 \%$ by $2015,0.5 \%$ by 2020, and finally $0 \%$ (phase-out) by 2030 .

But 2030 is still a long way to go and essential monitoring methods of refrigerant leakage must be adopted. Also, the effectiveness of the leakage detection depends on the present technologies available. Banning of refrigerants in simple steps cannot be achieved easily.

Although some developed countries like USA and Canada, which have already taken important and breakthrough steps in doing so. The developing countries have to travel an extra mile in doing so. Apart from ban of these refrigerants the financial burden to be faced by the industries in new installations must be kept in mind. Figure 17 shows an estimation of the ozone concentration in the earth's atmosphere with and without the Montreal protocol.

\section{VI.CONCLUSION}

The major problem faced in industrial and domestic refrigerators is the leakage of refrigerant. This leakage is main reason in the decrease in the performance of the refrigerator. The leakage may occur at the condenser, compressor, electronic expansion valve, or at the evaporator.

In this paper, we have expressed our concern on the damage caused by the refrigerants like CFCs and HCFs to the ozone layer [11] which may lead to serious consequences like skin cancer and also leakage of high quantities of these refrigerants is highly toxic and can be fatal. [12]

\section{REFERENCES}

[1] Siegfried Haaf, Helmut Henrici "Refrigeration Technology" in Ullmann'sEncyclopedia of Industrial Chemistry, 2002, WileyVCH, doi:10.1002/14356007.b03 19

[2] Handbook | ashrae.org https://www.ashrae.org/resources-publications/handbook

[3] Rosenthal, Elisabeth; Lehren, Andrew (June 20, 2011). "Relief in Every Window, but Global Worry Too". New York Times.Retrieved June 21, 2012.

[4] Of Montreal and Kyoto: A Tale of Two Protocols by Cass R. Sunstein 38 ELR 10566 8/2008

[5] "The Plain English Guide to the Clean Air Act" (PDF).

[6] "NRDC: Environmental Laws and Treaties" www.nrdc.org. Retrieved 2015-12-22.

[7] What is the phase-out schedule for HCFC refrigerants? - ashrae https://www.ashrae.org/File\%20Library/docLib/Technology/...TC02.05-FAQ-33.pdf

[8] THE REFRIGERATION SYSTEM - certify.ag/wpcontent/uploads/2016/03/Refrigeration_System.pdf

[9] Serial port Bluetooth Module (Master/Slave): HC-05 - ITEAD Wiki-https://www.itead.cc/wiki/Serial_Port_Bluetooth_Module_ (Master/Slave)_:_HC-05

[10] GSM/GPRS Module - Sparkfun https://www.sparkfun.com/ datasheets/Cellular\%20Modules/CEL-09533-User's\%20Ma...

[11] https://en.wikipedia.org/wiki/Ozone_depletion

[12] How can CFCs affect my health? https://toxtown.nlm. nih.gov/text_version/chemicals.php?id=9 


\section{BIOGRAPHIES}

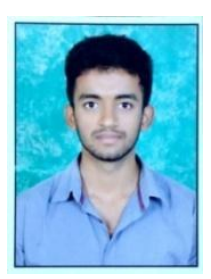

Mohan M who hails from Bangalore (Karnataka) is pursing B.E in Mechanical Engineering at RV College of Engineering, Bangalore. His area of interest in research is design and analysis of mechanical models and systems and aviation and aerospace.

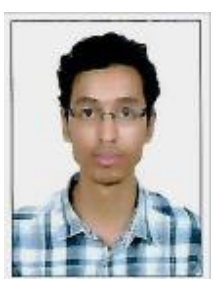

Vidyadhara BV who hails from Bangalore (Karnataka) is pursing B.E in Mechanical Engineering at RV College of Engineering, Bangalore. His area of interest in research is design and analysis of mechanical models and systems and aviation and aerospace.

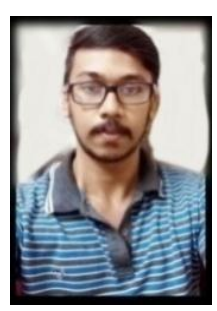

Vishesh $\mathbf{S}$ who hails from Bangalore (Karnataka) has completed B.E in Telecommunication Engineering from VTU, Belgaum, Karnataka in 2015. His research interest includes embedded systems, wireless communication and medical electronics. $\mathrm{He}$ is also the founder and director of the company Konigtronics (OPC) Pvt. Ltd.

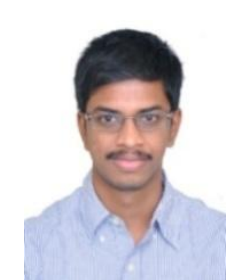

Manu Srinath hails from Bangalore (Karnataka); he has completed B.E in Telecommunication Engineering from VTU, Belgaum, Karnataka. His research interests include networking, image processing and cryptography. $\mathrm{He}$ is the Executive Officer at the company Konigtronics (OPC) Pvt. Ltd. 